\title{
Diseño y validación del Cuestionario para valorar la Carga Mental en los Deportes de Equipo (CCMDE)
}

\section{Design and validation of a Questionnaire to quantify the Mental Load in Team Sports (QMLST)}

\section{Desenho e validaçao de um Questionário para quantificar a Carga Mental em Equipes Esportivas (QCMEE)}

\author{
Díaz-García, J. ${ }^{1}$, González-Ponce, I. ${ }^{2}$, Ponce-Bordón, J.C. ${ }^{1}$, López-Gajardo, M.Á. ${ }^{1}$, García-Calvo, T. ${ }^{1}$ \\ ${ }^{1}$ Facultad de Ciencias del Deporte, Universidad de Extremadura (Cáceres, España); \\ ${ }^{2}$ Facultad de Educación, Universidad de Extremadura (Badajoz, España)
}

\begin{abstract}
RESUMEN
El objetivo del presente estudio fue diseñar y validar un cuestionario para valorar la carga mental en los deportes de equipo. Este cuestionario está formado por cuatro ítems en formato Likert (0-10): exigencia física, cognitiva, emocional y afectiva, y fue diseñado mediante acuerdo entre expertos $(n=10)$. Un total de 218 jugadores semiprofesionales de fútbol $(M=22.40 ; D T=2.25)$ completaron el cuestionario tras finalizar un entrenamiento. Se analizó la fiabilidad mediante Alfa de Cronbach y coeficiente de Omega. Además, para la validez concurrente se realizó un análisis de correlaciones bivariadas, utilizando la Escala Visual Analógica y la Escala de Percepción del Esfuerzo. Los resultados muestran que este cuestionario presenta una adecuada consistencia interna $(\alpha=.73 ; \omega=.75)$ $\mathrm{y}$ validez concurrente $(p<.05)$. Por tanto, es una herramienta válida y fiable que permitirá a los entrenadores $\mathrm{y}$ preparadores físicos valorar la carga mental específica en los deportes de equipo.
\end{abstract}

Palabras clave: Instrumento, validez, fiabilidad, fatiga mental, psicología deportiva

\begin{abstract}
The aim of the present study was to design and validate a questionnaire to quantify the mental load in team sports. Four items with Likert scale format (1-10) composed the instrument: physical, cognitive, emotional and affective exigence. A group of expert judges $(n=10)$ participated in the design. Later, a total of 218 semi-professionals soccer players $(M=22.40 ; \mathrm{S} D=2.25)$ answered the questionnaire after a training session. The internal consistency was evaluated with Cronbach's $\alpha(.73)$ and $\omega(.75)$. The concurrent validity was evaluated with Visual Analogue Scale's and Ratio Perceived Exertion's bivariate correlations $(p<.05)$. Thus, the present questionnaire is a reliability and adequate instrument for asses the specific mental load in team sports.
\end{abstract}

Keywords: Instrument, validity, reliability, mental fatigue, sport psychology. 


\section{Cuestionario Carga Mental Deportes Equipo}

\section{RESUMO}

O objetivo do presente estudo foi elaborar e validar um questionário para quantificar a carga mental em esportes coletivos. Quatro itens com formato de escala Likert (1-10) compuseram o instrumento: exigência física, cognitiva, emocional e afetiva. Um grupo de juízes especialistas $(n=10)$ participou do projeto. Um total de 218 jogadores semiprofissionais de futebol $(\mathrm{M}=22,40 ; \mathrm{DP}=2,25)$ responderam ao questionário após um treino. A consistência interna foi avaliada com $\alpha$ de Cronbach $(0,73)$ e $\omega(0,75)$. A validade concorrente foi avaliada com as correlações bivariadas da Escala Visual Analógica e Razão Percebida do Esforço ( $\mathrm{p}<0,05)$. Assim, o presente questionário é um instrumento confiável e adequado para avaliar a carga mental específica em esportes coletivos.

Palavras chave: Instrumento, validade, confiabilidade, fadiga mental, psicologia do esporte

\section{INTRODUCCIÓN}

Tradicionalmente, la cuantificación de la carga de entrenamiento en los deportes de equipo se ha centrado en los aspectos metabólicos y neuromusculares (Thompson et al., 2020). En los últimos años se ha podido observar un emergente interés por los aspectos psicológicos, habiendo quedado demostrado que la fatiga mental influye negativamente al rendimiento físico (Van Cutsem et al., 2017), técnico (Moreira et al., 2018; Smith et al., 2016) y táctico (Coutinho et al., 2017). Dentro de las metodologías de entrenamiento ecológicas se está tratando de valorar el efecto que distintos constreñimientos producen sobre la carga y fatiga mental, sin embargo, hasta el momento no existen instrumentos específicos para la cuantificación del esfuerzo mental que suponen las situaciones de entrenamiento o competición. El objetivo del presente estudio fue diseñar un instrumento específico para valorar la carga mental en los deportes de equipo.

La fatiga suele estar asociada a una disminución en el rendimiento deportivo, y puede estar causada tanto por aspectos físicos como psicológicos (Ishii, Tanaka, y Watanabe, 2014). Mientras que, habitualmente, la fatiga física produce una disfunción en los sistemas metabólico, neuromuscular o cardiorrespiratorio (Pageaux y Lepers, 2018), se ha podido comprobar que la fatiga mental no produce alteraciones en estos sistemas (Van Cutsem et al., 2017). Por lo tanto, el rol del cerebro parece evidente en la fatiga mental (Van Cutsem et al., 2020). La fatiga mental puede definirse como un estado psicobiológico causado por periodos prolongados de actividad mental exigente (Van Cutsem et al., 2017). En el contexto deportivo, se ha utilizado el término carga mental para definir la cantidad de esfuerzo mental necesario para desarrollar una tarea en un periodo de tiempo marcado, que está influido por aspectos físicos, cognitivos, afectivos y emocionales, (García-Calvo et al., 2019). Por tanto, aquellas situaciones deportivas que produzcan elevados niveles de carga mental (causa) podrían generar fatiga mental (consecuencia) en los deportistas. A nivel teórico, esto supone que los entrenadores pueden modificar los estímulos de entrenamiento para generar mayor o menor carga mental (por ejemplo, los ejercicios con mayores demandas cognitivas supondrían mayor carga mental) para generar una respuesta de fatiga mental mayor o menor en función de nuestros objetivos.

Como se comentó anteriormente, diversos estudios han demostrado que la fatiga mental puede afectar el rendimiento físico, técnico y táctico. En un estudio de revisión sistemática, Van Cutsem et al. (2017) definieron que los resultados obtenidos en estudios previos, en los que la fatiga mental reducía significativamente el volumen total de trabajo, pueden explicarse por un incremento en la percepción subjetiva del esfuerzo. Este fenómeno parece estar causado por la acumulación de adenosina en el cerebro (Martin et al., 2019), y también explica los efectos negativos que la fatiga mental produce en el comportamiento táctico. Coutinho et al. (2017), reportaron una reducción en las distancias entre jugadores de futbol después de completar una tarea mental exigente. Estos autores sugieren que los incrementos en la sensación de fatiga física disminuyen la confianza de los jugadores para obtener el rendimiento óptimo en determinadas acciones, lo que lleva a los jugadores a reducir la distancia entre líneas del equipo (al estar más cerca de los compañeros, es más fácil realizar ayudas). Esta respuesta táctica colectiva empeora la ocupación de los espacios, lo que puede afectar negativamente al rendimiento. Respecto a la disminución de la eficacia 


\section{Díaz-García et al.}

en las acciones técnicas, Peageaux y Lepers (2018) definen que la fatiga mental produce un empeoramiento en el rendimiento cognitivo, que aumenta el número de errores en acciones técnicas. Por tanto, parece evidente la necesidad de cuantificar la carga mental que se producen en las situaciones de entrenamiento y competición, con la finalidad de evitar la aparición de la fatiga mental.

Trabajos anteriores han tratado de definir variables biológicas para determinar de manera objetiva los niveles de carga y fatiga mental, a través de la pupilometría o las alteraciones electroencefalográficas (Fink et al., 2018). Sin embargo, la mayor parte de estas técnicas no pueden ser utilizadas en acciones específicas de los deportes de equipo, dado que la mayoría tienen que ser utilizadas en reposo. Por ello, han prosperado el uso de instrumentos, con una mayor aplicación en contextos con alta validez ecológica, como son las escalas y los cuestionarios. Entre ellas, la más utilizada ha sido el NASA TXL, pero la poca especificidad de alguno de los ítems de estos cuestionarios podría provocar una pérdida de validez ecológica y/o aplicación en las mediciones (Thompson et al., 2019). Según Van Cutsem et al. (2017) el esfuerzo mental en el contexto deportivo está producido por aspectos cognitivos y emocionales. La carga cognitiva hace referencia a (i) situaciones que implican altos niveles de concentración y atención; (ii) el procesamiento de la información contextual en base a la cual se toman las decisiones (táctica); y (iii) la dificultad en la comprensión de las tareas (GarcíaCalvo, 2017). Por su parte, los aspectos emocionales incluyen la afectividad e interdependencia en las relaciones entre las personas que participan (Teques, Duarte, y Viana, 2019), pero también las emociones específicas que se producen durante el desarrollo de las tareas, como el afrontamiento de responsabilidades o la consecución de los objetivos (Thompson et al., 2020). Por último, la carga física específica de cada tarea también podría influir en la carga mental (Van Cutsem et al., 2017). En consecuencia, parece que todos estos ítems deben ser tenidos en cuenta a la hora de cuantificar la carga mental en los deportes de equipo.

Por tanto, a pesar del creciente interés que se les ha otorgado a los aspectos mentales en los deportes de equipo, la falta de instrumentos específicos para su cuantificación provoca que los autores continúen recurriendo a instrumentos genéricos, que no tienen en cuenta las particularidades ni la multifactorialidad de los procesos mentales en este tipo de deportes. Por ello, el objetivo del presente estudio fue diseñar y validar un cuestionario que atendiese a las características específicas para la valoración de la carga mental en los deportes de equipo.

\section{MATERIAL Y MÉTODOS}

\section{Procedimiento}

El presente estudio fue aprobado por el Comité de Bioética de la Universidad de Extremadura (93/2020). Todos los datos han sido procesados acorde a las recomendaciones de ética y privacidad de la Asociación Americana de Psicología (2019).

El objetivo de la primera parte del estudio fue el diseño y desarrollo del instrumento. Para ello, se elaboró una consulta a un grupo de 10 expertos en Psicología y Ciencias del Deporte. La creación de un comité de expertos para el desarrollo de un instrumento es una práctica muy común, por el conocimiento que tienen los mismos de la temática y las aportaciones que pueden realizar. Además, esta metodología está justificada desde el punto de vista científico (Valles, 2003). Los expertos consultados cumplían los siguientes requisitos: (i) ser graduados en Ciencias del Deporte y/o Psicología; (ii) ser doctor, es decir, haber defendido con éxito la tesis doctoral en el momento que comenzó la investigación; y (iii) tener publicaciones científicas de impacto recientes en el ámbito de la psicología de los deportes de equipo.

Para esta parte del estudio, se concretaron una serie de reuniones. En una primera reunión con el grupo, los expertos explicaron en qué consistía para ellos la carga mental en los deportes de equipo, y propusieron distintos ítems y constructos que consideraban interesantes para la cuantificación de la carga mental. En una reunión posterior, con una semana de diferencia con respecto a la primera, se realizó una puesta en común, y a través de un consenso se seleccionaron los ítems que mejor analizaban los contenidos a valorar. Finalmente, se acordó que los cuatro ítems: exigencia física, cognitiva, emocional y afectiva, eran representativos de la carga mental en los deportes de equipo, según el juicio de los expertos mencionados.

A continuación, la segunda fase del estudio tenía como objetivo la puesta en práctica y el análisis psicométrico 


\section{Cuestionario Carga Mental Deportes Equipo}

de las propiedades del instrumento para su validación. El investigador principal se puso en contacto con los responsables de los equipos del grupo XIV de la Tercera División Nacional y sus entrenadores. Los participantes aceptaron su participación, fueron informados del objetivo del estudio y firmaron un consentimiento informado.

Los jugadores participantes, asistieron a una charla previa en la que se les explico el significado concreto de cada ítem, asegurando su comprensión previamente a contestar el instrumento. No se realizó ninguna modificación de los ítems como consecuencia de las dudas de los jugadores. Finalmente, los participantes rellenaron el cuestionario, de forma individual a la finalización de un entrenamiento. La duración total de las respuestas fue aproximadamente de cinco minutos, y se produjo en un clima adecuado para que permitiese a los jugadores concentrarse sin distracciones. Además, también fueron rellenados el RPE y el VAS.

\section{Participantes}

La muestra del estudio estuvo conformada por 218 jugadores masculinos semiprofesionales de fútbol, con edades comprendidas entre los 18 y los 36 años $(M=$ 24.81; $D T=3.51)$. Los participantes competían en el grupo XIV de la Tercera División Nacional de fútbol, durante la temporada 2018/2019, y todos ellos contaban con una experiencia mínima de 14 años practicando fútbol.

\section{Instrumentos}

Cuestionario para la Valoración de la Carga Mental en los Deportes de Equipo (CCMDE; Díaz-García et al.). Este instrumento está conformado por cuatro ítems: exigencia física (¿Cómo de exigente cuantificarías el esfuerzo físico de esta sesión?), cognitiva (¿Cómo de exigente cuantificarías el esfuerzo cognitivo de esta sesión?), emocional (¿Cómo de exigente cuantificarías el esfuerzo realizado para manejar tus emociones durante esta sesión?) y afectiva (¿Cómo de exigente cuantificarías el esfuerzo realizado en esta sesión para manejar las relaciones emocionales con el resto de participantes?); en modalidad Escala Likert con rango de respuesta $0-10$, donde 0 significa nada y 10 es el valor máximo de exigencia para cada ítem.

VAS (Smith et al., 2017). Para valorar la fatiga mental, se les preguntaba a los deportistas “¿cómo de fatigado te sientes mentalmente en una línea de 0 a 100 ?”. Este instrumento se presenta en formato de escala, donde 0 es el mínimo y 100 el máximo. El uso del VAS para valorar la fatiga mental en los deportes de equipo ha sido utilizado en trabajos previos (e.g., García-Calvo et al. 2019). Aunque no se ha encontrado ningún estudio en el que se desarrolle una validación para el uso de este instrumento en la valoración de la fatiga mental en el futbol, para algunas variables que guardan relación con la fatiga mental, como la ansiedad (Facco et al, 2013) o la fatiga general (Jollant et al., 2019), sí se han encontrado estudios que analizan las propiedades psicométricas del instrumento.

RPE (Costa et al., 2019). Con la finalidad de valorar la percepción de esfuerzo, se le pidió al deportista "indicar el nivel de esfuerzo físico que percibe que ha tenido la tarea para sí mismo en términos de 0 a 10", donde 0 es Reposo y 10 es Máximo. El uso de este instrumento para valorar la carga de entrenamiento en deportes de equipo fue justificado por Costa et al. (2019), quienes concluyen que el RPE es un instrumento válido para cuantificar la carga y fatiga de entrenamiento en deportes colectivos.

\section{Análisis estadístico}

Para el tratamiento y análisis de los datos se utilizó el programa estadístico SPSS 25.0 (IBM, 2017). En primer lugar, se realizó un análisis factorial exploratorio (AFE) con el objetivo de estudiar la validez de constructo. Además, se calculó la fiabilidad a través de Alfa de Cronbach (Cronbach, 1990), utilizando como valor de referencia el valor .70 (Field, 2009; Polit y Hungler, 2000), y coeficiente de Omega. Por otra parte, se llevó a cabo un análisis de correlaciones bivariadas, con el objetivo de probar la validez concurrente del instrumento, utilizando los instrumentos RPE y VAS para este procedimiento.

\section{RESULTADOS}

En la Tabla 1 se muestran los pesos factoriales entre los diferentes ítems del cuestionario. Como se puede observar, los ítems del cuestionario están agrupados en torno a un único factor, que en este caso es la carga mental. En consecuencia, no fue necesario realizar un análisis factorial confirmatorio. 


\section{Díaz-García et al.}

\section{Tabla 1}

Pesos factoriales entre items

\begin{tabular}{lc}
\hline Ítems & Pesos de regresión \\
Ítem 1. Exigencia física & .81 \\
Ítem 2. Exigencia cognitiva & .72 \\
Ítem 3. Exigencia emocional & .70 \\
Ítem 4. Exigencia afectiva & .67
\end{tabular}

A continuación, en la Tabla 2 se muestran los estadísticos descriptivos de las puntuaciones obtenidas para los ítems del instrumento y el análisis de correlaciones bivariadas entre ítems. Los resultados sugieren que existe relación significativa entre ítems del instrumento, pero estos no llegan a superponerse $(>.80)$, lo cual hubiese supuesto una reunificación de los mismos.

\section{Tabla 2}

\section{Estadísticos descriptivos y correlaciones bivariadas} entre ítems del instrumento

\begin{tabular}{lll}
\hline Ítems & $M$ & $D T$ \\
Ítem 1. Exigencia física & 6.67 & 1.95 \\
Ítem 2. Exigencia cognitiva & 6.71 & 1.97 \\
Ítem 3. Exigencia emocional & 6.19 & 2.63 \\
Ítem 4. Exigencia afectiva & 5.75 & 2.74
\end{tabular}

Nota. ${ }^{*} p<.05 .1$ 1= Exigencia física; $2=$ Exigencia cognitiva; 3 = Exigencia emocional; $4=$ Exigencia afectiva

Finalmente, en la Tabla 3 se pueden observar los resultados para la validez concurrente, calculado mediante el análisis de correlaciones bivariadas entre el CCMDE, el RPE y el VAS. Los resultados muestran relaciones significativas entre el CCMDE y los dos instrumentos utilizados, confirmando así la validez concurrente del instrumento. Además, también queda confirmada la fiabilidad del instrumento en base a los resultados $\alpha$ y $\omega$.

\section{Tabla 3}

Estadísticos descriptivos y correlaciones con RPE y VAS

\begin{tabular}{llllllll}
\hline Ítems & $M$ & $D T$ & $\alpha$ & $\omega$ & 1 & 2 & 3 \\
CCMDE & 6.33 & 1.95 & .73 & .75 & - & - & - \\
RPE & 5.32 & 1.97 & - & - & $.44^{*}$ & - & - \\
VAS & 36.2 & 23.66 & - & - & $.45^{*}$ & $.57^{*}$ & - \\
\hline
\end{tabular}

Nota. ${ }^{*} p<.05 .1=$ CCMDE; 2 = RPE; 3 = VAS.

\section{DISCUSIÓN}

El objetivo principal del estudio fue diseñar y probar la validez y fiabilidad del CCMDE para cuantificar la carga mental en los deportes de equipo. En base a los resultados obtenidos, el CCMDE es un instrumento específico, válido y fiable para el propósito descrito.

Para el análisis de la validez concurrente, se utilizó la correlación entre el CCMDE, el VAS y el RPE, dos de las escalas más utilizadas para valorar la carga y la

1 fatiga ę los deportes ae equipo en latactualidad. Los resultados muestran una correlación significativa entre

- estos instrumentos y el CCMDE. La escala VAS ha sido utilizada principalmente para cuantificar la fatiga

.54光ental en el deporte. Previos estudios han definido los efectos que causan la realización de tareas .33 hiventalmtefte exigentes sobre la fatiga mental. En todos ellos, se pudo observar que la fatiga mental se

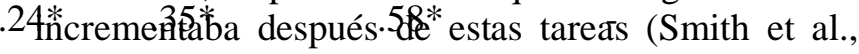
2016; Van Cutsem et al, 2020). Por tanto, la relación significativa entre el CCMDE y el VAS parece justificada, en cuanto a que la carga mental sería la causante de la fatiga mental.

Por otro lado, el RPE ha sido utilizado principalmente para conocer el esfuerzo físico percibido por los deportistas después de una tarea o competición (Calahorro-Cañada, Torres-Luque y Lara-Sánchez, 2014). Estos resultados sugieren una relación significativa entre el esfuerzo físico percibido y la carga mental. Esta relación ya había sido previamente definida por Van Cutsem et al. (2017), quienes 


\section{Cuestionario Carga Mental Deportes Equipo}

explicaban que las tareas con mayor carga mental y, especialmente, la aparición de la fatiga mental genera un incremento en el esfuerzo físico percibido. Martin et al. (2018) explican que la acumulación de adenosina extracelular en el cerebro sería la causante de esta relación.

Respecto al análisis de la fiabilidad, se confirma el alto grado de acuerdo entre los ítems del CCMDE (Field, 2009; Polit y Hungler, 2000). Como se definió en la introducción, la influencia de los aspectos físicos, cognitivos, emocionales y afectivos sobre la carga y fatiga mental había sido previamente testada (Coutinho et al., 2017; Pageaux y Lepers, 2018; Van Cutsem et al., 2017). Para el diseño del instrumento, se utilizó una muestra de expertos sobre la temática, que conocen en profundidad las características de la carga mental. Probablemente, este conocimiento del constructo estudiado haya llevado a obtener estos adecuados resultados de fiabilidad.

\section{CONCLUSIONES}

Por tanto, en base a los resultados encontrados, se puede concluir que el CCMF cumple los criterios mínimos, establecidos por la comunidad científica, de fiabilidad y validez para poder ser empleado para la valoración de la carga mental en los deportes de equipo atendiendo a las especificidades de este constructo en el contexto descrito.

\section{APLICACIONES PRÁCTICAS}

Respecto a las principales aplicaciones prácticas que permite el CCMDE, nos gustaría destacar: (i) el breve número de ítems que presenta, lo cual permite involucrar la valoración de la carga mental en los entrenamientos y planificaciones, sin tener que esperar varios días a recibir los resultados y sin restar tiempo efectivo de entrenamiento o post-entrenamiento a los deportistas, (ii) su especificidad, puesto que no se han encontrado otros instrumentos que se hayan validado de forma exclusiva para valorar la carga mental en el fútbol (Russell, Jenkins, Smith, Halson, \& Kelly, 2019; Thompson et al., 2020), y (iii) el bajo coste del instrumento.

En primer lugar, la presencia de únicamente cuatro ítems permite involucrar a este instrumento en los entrenamientos y en las dinámicas semanales de los equipos de competición, favoreciendo que los entrenadores y preparadores físicos realicen adaptaciones en la carga de entrenamiento o tomen decisiones acordes a sus objetivos, teniendo en cuenta también los niveles de carga mental (García-Calvo, 2017). Las teorías de la planificación en los deportes de equipo han evolucionado a lo largo del tiempo, principalmente por la necesidad de rendir de forma óptima cada semana (Buchheit, Lacome, Cholley y Simpson, 2018). Esto ha provocado que la preparación física, el trabajo técnico-táctico y los aspectos psicológicos formen parte de un conjunto único dentro de las metodologías de entrenamiento y, por tanto, es necesario controlar y cuantificar todos estos aspectos (Nedelec et al., 2012; Sánchez-Sánchez et al., 2014). La presencia del CCMDE, con sus características, puede solucionar la problemática actual para cuantificar la carga mental de forma específica, rápida y con extracción inmediata de los resultados, además de constituirse como una herramienta accesible para cualquier equipo, independientemente de la categoría o nivel económico del mismo.

\section{AGRADECIMIENTOS}

Este trabajo tuvo el soporte de las ayudas a grupos (GR18102) de la Junta de Extremadura (Ministerio de Empleo e Infraestructuras) con la contribución de la Unión Europea a través de los Fondos de Desarrollo Regional. Además, este trabajo está sustentado por un contrato predoctoral del Programa de Formación del Profesorado Universitario del Gobierno de España (FPU18/03660).

\section{REFERENCIAS}

1. American Psychological Association (2019). APA Publication Manual of the American Psychological Association (7a ed.). Washington: autor.

2. Buchheit, M., Lacome, M., Cholley, Y., y Simpson, B. M. (2018). Neuromuscular responses to conditioned soccer sessions assessed via GPSEmbedded accelerometers: Insights into tactical periodization. International Journal of Sports Physiology and Performance, 13(5), 577-583. https://doi.org/10.1123/ijspp.2017-0045

3. Calahorro-Cañada, F., Torres-Luque, G., y LaraSánchez, A. (2014). La percepción subjetiva de esfuerzo como herramienta válida para la monitorización de la intensidad del esfuerzo en competición de jóvenes futbolistas. Cuadernos de Psicología del Deporte, 14(1), 75-82. 


\section{Díaz-García et al.}

4. Coutinho, D., Gonçalves, B., Travassos, B., Wong, D. P., Coutts, A. J., y Sampaio, J. E. (2017). Mental fatigue and spatial references impair soccer players' physical and tactical performances. Frontiers in Psychology, 8, 16451653. https://doi.org/10.3389/fpsyg.2017.01645

5. Costa, J. A., Brito, J., Nakamura, F. Y., Figueiredo, P., y Rebelo, A. (2019). Using the rating of perceived exertion and heart rate to quantify training intensity in female soccer players: Validity and utility. Journal of Strength and Conditioning Research, in press. https://doi.org/10.1519/JSC.0000000000003407

6. Cronbach, L.J. (1990). Essentials of psychological testing (5th Ed.). New York: Harper \& Row.

7. Facco, E., Stellini, E., Bacci, C., Manani, G., Pavan, C., Cavallin, F., y Zanette, G. (2013). Validation of visual analogue scale for anxiety (VAS-A) in preanesthesia evaluation. Minerva Anestesiológica, 79(12), 1389-1395.

8. Field, A. (2009). Discovering statistics using SPSS. Los Angeles: Sage publications.

9. Fink, A., Rominger, C., Benedek, M., Perchtold, C. M., Papousek, I., Weiss, E. M., Seidel, A., y Memmert, D. (2018). EEG alpha activity during imagining creative moves in soccer decisionmaking situations. Neuropsychologia, 114, 118124.https://doi.org/10.1016/j.neuropsychologia.2 018.04 .025

10. García Calvo, T. (2017). The mental load of training in football: Conceptual aspects for its assessment and training. Revista de Preparación Física en el Fútbol, 24, 23-33.

11. García-Calvo, T., González-Ponce, I., Ponce, J. C., Tomé-Lourido, D., y Vales-Vázquez, Á. (2019). Incidence of the tasks scoring system on the mental load in football training. Revista de Psicología del Deporte, 28(2), 79-86.

12. IBM SPSS Statistics for Windows, Version 25.0. Armonk, NY: IBM Corp

13. Ishii, A., Tanaka, M., y Watanabe, Y. (2014). Neural mechanisms of mental fatigue. Reviews in the Neurosciences, 25(4), 469-479. https://doi.org/10.1515/revneuro-2014-0028

14. Jollant, F., Voegeli, G., Kordsmeier, N. C., Carbajal, J. M., Richard-Devantoy, S., Turecki, G., y Cáceda, R. (2019). A visual analog scale to measure psychological and physical pain: A preliminary validation of the PPP-VAS in two independent samples of depressed patients.
Progress in Neuro-psychopharmacology \& Biological Psychiatry, 90, 55-61. https://doi.org/10.1016/j.pnpbp.2018.10.018

15. Martin, K., Meeusen, R., Thompson, K. G., Keegan, R., y Rattray, B. (2018). Mental fatigue impairs endurance performance: A physiological explanation. Sports medicine (Auckland, N.Z.), 48(9), 2041-2051. https://doi.org/10.1007/s40279-018-0946-9

16. Moreira, A., Aoki, M. S., Franchini, E., da Silva Machado, D. G., Paludo, A. C., y Okano, A. H. (2018). Mental fatigue impairs technical performance and alters neuroendocrine and autonomic responses in elite young basketball players. Physiology \& Behavior, 196, 112-118. https://doi.org/10.1016/j.physbeh.2018.08.015

17. Nédélec, M., McCall, A., Carling, C., Legall, F., Berthoin, S., y Dupont, G. (2012). Recovery in soccer: part I - post-match fatigue and time course of recovery. Sports Medicine (Auckland, N.Z.), 42(12), 997-1015. https://doi.org/10.2165/11635270-000000000$\underline{00000}$

18. Pageaux, B., y Lepers, R. (2018). The effects of mental fatigue on sport-related performance. Progress in brain research, 240, 291-315.

https://doi.org/10.1016/bs.pbr.2018.10.004

19. Polit, D., y Hungler, B. (2000). Investigación científica en Ciencias de la Salud ( $6^{\mathrm{a}}$ ed.). México: McGraw-Hill.

20. Russell, S., Jenkins, D., Smith, M., Halson, S., y Kelly, V. (2019). The application of mental fatigue research to elite team sport performance: New perspectives. Journal of Science and Medicine in Sport, 22(6), 723-728. https://doi.org/10.1016/j.jsams.2018.12.008

21. Sánchez-Sánchez, J., Luis-Pereira, J.M., GuillenRodríguez, J., Martín-García, D., y Romo-Martín, D. (2014). Efecto de la motivación del entrenador sobre la carga interna y el rendimiento físico de un juego de fútbol reducido $3 \times 3$. Cuadernos de Psicología del Deporte, 14(3), 169-176.

22. Smith, M. R., Coutts, A. J., Merlini, M., Deprez, D., Lenoir, M., y Marcora, S. M. (2016). Mental Fatigue Impairs Soccer-Specific Physical and Technical Performance. Medicine and Science in Sports and Exercise, 48(2), 267-276. https://doi.org/10.1249/MSS.0000000000000762 


\section{Cuestionario Carga Mental Deportes Equipo}

23. Smith, M. R., Fransen, J., Deprez, D., Lenoir, M., y Coutts, A. J. (2017). Impact of mental fatigue on speed and accuracy components of soccer-specific skills. Science and Medicine in Football, 1, 48-52. https://doi.org/10.1080/02640414.2016.1252850

24. Teques, P., Duarte, D., y Viana, J. (2019). Coaches' emotional intelligence and reactive behaviors in soccer matches: Mediating effects of coach efficacy beliefs. Frontiers in Psychology, 10 ,

1629. https://doi.org/10.3389/fpsyg.2019.01629

25. Thompson, C. J., Fransen, J., Skorski, S., Smith, M. R., Meyer, T., Barrett, S., y Coutts, A. J. (2019). Mental fatigue in football: Is it time to shift the goalposts? An evaluation of the current methodology. Sports Medicine (Auckland, N.Z.), 49(2), 177-183. https://doi.org/10.1007/s40279018-1016-Z

26. Thompson, C. J., Noon, M., Towlson, C., Perry, J., Coutts, A. J., Harper, L. D., ..., y Meyer, T. (2020). Understanding the presence of mental fatigue in English academy soccer players. Journal of Sports Sciences, 38(13), 1524-1530. https://doi.org/10.1080/02640414.2020.1746597
27. Valles, M. (2003). Técnicas cualitativas de investigación social: Reflexión metodológica y práctica profesional. Madrid: Editorial Síntesis.

28. Van Cutsem, J., Marcora, S., De Pauw, K., Bailey, S., Meeusen, R., y Roelands, B. (2017). The effects of mental fatigue on physical performance: A Systematic review. Sports Medicine (Auckland, N.Z.), 47(8), 1569-1588. https://doi.org/10.1007/s40279-016-0672-0

29. Van Cutsem, J., Roelands, B., Pluym, B., Tassignon, B., Verschueren, J. O., DE Pauw, K., y Meeusen, R. (2020). Can creatine combat the mental fatigue-associated decrease in visuomotor skills?. Medicine and science in sports and exercise, 52(1), $120-130$. https://doi.org/10.1249/MSS.0000000000002122 